\title{
Elevated LRRK2 autophosphorylation in brain-derived and peripheral exosomes in LRRK2 mutation carriers
}

\author{
Shijie Wang ${ }^{1}$, Zhiyong Liu' ${ }^{1}$, Tao Ye², Omar S. Mabrouk², Tyler Maltbie', Jan Aasly ${ }^{3^{*}}$ and Andrew B. West ${ }^{1 *}$
}

\begin{abstract}
Missense mutations in the leucine-rich repeat kinase 2 (LRRK2) gene can cause late-onset Parkinson disease (PD). LRRK2 mutations increase LRRK2 kinase activities that may increase levels of LRRK2 autophosphorylation at serine 1292 (pS1292) and neurotoxicity in model systems. pS1292-LRRK2 protein can be packaged into exosomes and measured in biobanked urine. Herein we provide evidence that PS1292-LRRK2 protein is robustly expressed in cerebral spinal fluid (CSF) exosomes. In a novel cohort of Norwegian subjects with and without the G2019S-LRRK2 mutation, with and without PD, we quantified levels of pS1292-LRRK2, total LRRK2, and other exosome proteins in urine from 132 subjects and in CSF from 82 subjects. CSF and urine were collected from the same morning clinic visit in 55 of the participants. We found that total LRRK2 protein concentration was similar in exosomes purified from either CSF or urine but the levels did not correlate. pS1292-LRRK2 levels were higher in urinary exosomes from male and female subjects with a LRRK2 mutation. Male LRRK2 mutation carriers without PD had intermediate pS1292-LRRK2 levels compared to male carriers with PD and controls. However, female LRRK2 mutation carriers without PD had the same pS1292-LRRK2 levels compared to female carriers with PD. pS1292-LRRK2 levels in CSF exosomes were near saturated in most subjects, ten-fold higher on average than pS1292-LRRK2 levels in urinary exosomes, irrespective of LRRK2 mutation status or PD diagnosis. These results provide insights into the effects of LRRK2 mutations in both the periphery and brain in a well-characterized clinical population and show that LRRK2 protein in brain exosomes may be much more active than in the periphery in most subjects.
\end{abstract}

Keywords: Park8, dardarin, Neurodegeneration, Movement Disorders, Biomarkers, Microvesicles

\section{Background}

Genetic variation in the leucine-rich repeat kinase 2 (LRRK2) gene associates with Parkinson disease (PD) susceptibility [21, 34]. Rare pathogenic missense mutations in exons encoding the GTPase domain (termed ROC) and linking COR (C-terminal of ROC) domains strongly predispose to late-onset PD [29]. The more common mutation in the kinase domain that may be present in $0.1 \%$ of Western populations, G2019S, has more variable and typically lower penetrance [32]. Lifetime risk for PD in G2019S carriers may be $80 \%$ in

\footnotetext{
* Correspondence: jan.aasly@ntnu.no; abwest@uab.edu

${ }^{3}$ Dept of Neurology, Dept of Neuroscience, St. Olavs Hospital, Norwegian University of Science and Technology, 7030 Trondheim, Norway

${ }^{1}$ Department of Neurology, Center for Neurodegeneration and Experimental Therapeutics, University of Alabama at Birmingham, Birmingham, AL 35233, USA

Full list of author information is available at the end of the article
}

North African Berbers but as low as $20-30 \%$ in Ashkenazi Jews $[17,20,29]$. Studies analyzing $A \beta$, tau, and $\alpha-$ synuclein proteins in cerebral spinal fluid (CSF) from LRRK2 mutations carriers have identified nominal differences compared to idiopathic PD [1, 30]. Genome-wide association studies have identified common polymorphisms in LRRK2 that associate with idiopathic PD, implicating LRRK2 function in susceptibility to late-onset PD in individuals without pathogenic mutations [19]. Few studies have yet biochemically analyzed LRRK2 protein in clinical samples from individuals with LRRK2 mutations or in the general idiopathic PD population.

In model systems, pathogenic LRRK2 mutations increase the proportion of protein that is autophosphorylated [33], particularly at the serine 1292 residue [23]. We and others have demonstrated that LRRK2 kinase activity, and autophosphorylation at the 1292 residue, is 
required for LRRK2-linked neurotoxicity [11, 25, 26, 33]. The high frequency of $L R R K 2$ mutations in PD, particularly the G2019S mutation with incomplete penetrance, presents a unique opportunity to understand how surrogates of LRRK2 kinase activity like autophosphorylation may predict and drive PD progression [2, 13]. Previously, we demonstrated in urinary exosomes that the ratio of phosphorylated pS1292-LRRK2 to total LRRK2 is increased in male G2019S-LRRK2 mutation carriers of Ashkenazi Jewish descent [6]. However, in vitro evidence suggests LRRK2 kinase activity stabilizes LRRK2 expression so that the ratio of pS1292-LRRK2 to total LRRK2 may not capture the overall increase in pS1292-LRRK2 protein [24]. Indeed, in a larger sample series of idiopathic PD from Birmingham, Alabama, we found that pS1292LRRK2 levels are closely correlated with total LRRK2 levels when normalized to exosome housekeeping proteins [8]. Several studies have demonstrated increases in LRRK2 protein in frontal cortex post-mortem tissue from idiopathic PD and control $[4,5]$. There are no previous studies evaluating pS1292-LRRK2 protein in the brain in clinical populations or in post-mortem studies. As LRRK2 kinase inhibitors move forward to the clinic, the characterization of kinase-activity surrogates like pS1292LRRK2 in the brain may help identify subjects most likely to benefit from reductions of LRRK2 kinase activity [32].

Here, we quantify the absolute amount of pS1292LRRK2 in CSF and urine from a novel cross-sectional cohort collected exclusively in Trondheim, Norway, focusing on G2019S-LRRK2 mutation carriers. We measured pS1292-LRRK2, total LRRK2, and housekeeping exosomal proteins in both urine and CSF collected between 8 and 9:00 AM in (overnight) fasting Norwegians. Male and female subjects with or without PD, with or without the G2019S-LRRK2 mutation, were evaluated. We found elevated pS1292-LRRK2 levels in urinary exosomes from mutation carriers versus controls. Strikingly, pS1292-LRRK2 levels were near saturated in most $L R R K 2$ mutation carriers in exosomes isolated from the
CSF. These results provide the first measurements of brain-derived LRRK2 protein in clinical samples. While we could not distinguish LRRK2 mutation carriers from controls in CSF due to saturation of the pS1292-LRRK2 phosphorylation site in many of the subjects, our results further link the G2019S-LRRK2 mutation with upregulated kinase activity.

\section{Methods}

\section{Clinical cohort and biospecimens}

All protocols were approved by the local Institutional Review Boards. Participants were recruited in the outpatient clinic of the Department of Neurology, St. Olavs hospital, Trondheim, Norway. Urine specimens were collected for this study from 132 participants that included healthy controls (LRRK2- PD-), age-matched G2019S-LRRK2 mutation carriers without manifest PD (LRRK2+ PD-), G2019SLRRK2 mutation carriers with PD (LRRK2 + PD+), and non-LRRK2 mutation carriers with PD (i.e., idiopathic PD, LRRK2- PD+, see Table 1). CSF specimens were collected from 82 participants (Table 2). Of these subjects, 55 donated both urine and CSF in the same clinic visit and were analyzed here (Table 3). All lumbar punctures were performed between 8 and $9 \mathrm{AM}$ on overnight fasting subjects, and urine samples were collected thereafter to around 10 AM. A small amount of CSF was immediately sent for cell counts and tests for glucose and proteins. Urine and CSF was centrifuged within 15 minutes after the end of collection, aliquoted, and biobanked.

Urine and CSF samples were processed and analyzed by an investigator blinded to their identify. CSF hemoglobin level was determined using ELISA method (Bethyl Labs, \#E88-134). Urine was assessed for leukocyte count, $\mathrm{pH}$, glucose, total protein, red-blood cells, and specific gravity via test strips (Fisher). All participants were clinically evaluated for PD severity using the Unified Parkinson's Disease Rating Scale (UPDRS) part III, Hoehn \& Yahr scale, modified Schwab \& England scale, Montreal Cognitive Assessment (MoCA),

Table 1 Clinical data and demographics corresponding to urine samples from subjects with or without G2019S-LRRK2 mutations, with or without PD

\begin{tabular}{|c|c|c|c|c|c|}
\hline & LRRK2+/PD- & LRRK2+/PD+ & LRRK2-/PD+ & LRRK2-/PD- & $p$ value $^{a}$ \\
\hline Gender M(F) & $29(30)$ & $8(18)$ & $23(16)$ & $2(6)$ & ns \\
\hline Age & $60(52-66)$ & $59(53.75-70.25)$ & $59(53-63)$ & $60.5(56-75)$ & ns \\
\hline Age at onset & NA & $55(47-60.25)$ & $55(50-60)$ & NA & ns \\
\hline LEDD & NA & $500(400-650)$ & $300(0-600)$ & NA & ns \\
\hline UPDRS_III & $4(0-6)$ & $19.5(15.75-22.25)$ & $19(16.5-24)$ & $0(0-0)$ & $<0.0001$ \\
\hline MoCA & $26(26-29)$ & $26(25-27)$ & $28(26-28)$ & $27(<26-30)$ & ns \\
\hline$H \& Y$ & $0(0-0)$ & $2(2-2.5)$ & $2(2-2)$ & $0(0-0)$ & $<0.0001$ \\
\hline
\end{tabular}

LEDD is L-dopa equivalent daily dosage, UPDRS is Unified Parkinson's Disease Rating Scale, MoCA is Montreal Cognitive Assessment, H\&Y is Hoehn \& Yahr scale, NA

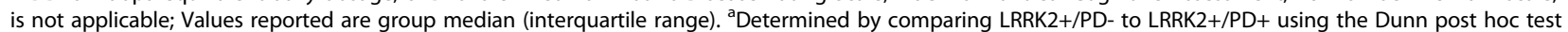
following Kruskal-Wallis test of all groups. $n s$ is not significant as determined by Pearson chi square (gender and MoCA), ANOVA (age at test), $t$ test (age at onset), Mann-Whitney test (LEDD) and Kruskal-Wallis test (H\&Y, UPDRS III) 
Table 2 Clinical data and demographics corresponding to CSF samples from subjects with or without G2019S-LRRK2 mutations, with or without PD

\begin{tabular}{llllll}
\hline & LRRK2+/PD- & LRRK2+/PD+ & LRRK2-/PD+ & LRRK2-/PD- & $p$ value \\
\hline Gender M(F) & $13(26)$ & $3(16)$ & $11(8)$ & $0(5)$ & 0.008 \\
Age & $63(56-69)$ & $57(53-69)$ & $60(50-67)$ & $60(44.5-74)$ & NA \\
Age at onset & NA & $55(47-65)$ & $57(50-60)$ & NA & ns \\
LEDD & NA & $600(250-675)$ & $300(0-550)$ & $0(0-0)$ & ns \\
UPDRS_III & $5(0-6)$ & $18(15-22)$ & $20(16.25-22)$ & $27(<26-30)$ & 0.0001 \\
MoCA & $26(26-28)$ & $26(25-27)$ & $2(2-2)$ & $0(0-0)$ & ns
\end{tabular}

LEDD is L-dopa equivalent daily dosage, UPDRS is Unified Parkinson's Disease Rating Scale, MoCA is Montreal Cognitive Assessment, H\&Y is Hoehn \& Yahr scale, NA is not applicable; Values reported are group median (interquartile range). ${ }^{a}$ Determined by comparing LRRK2+/PD- to LRRK2+/PD+ using the Dunn post hoc test following Kruskal-Wallis test of all groups. $n$ s is not significant as determined by Pearson chi square (MoCA), ANOVA (age at test), $t$ test (age at onset), Mann-Whitney test (LEDD) and Kruskal-Wallis test (H\&Y, UPDRS III)

and L-dopa equivalent daily dose (LEDD) calculated as described [8].

\section{Exosome isolation and characterization}

Supernatants from cell cultures were centrifuged 10,000 $\mathrm{x} \mathrm{g}$ at $4{ }^{\circ} \mathrm{C}$ for $30 \mathrm{~min}$, supernatant and centrifuged at $100,000 \times \mathrm{g}$ for 1 hour at $4^{\circ} \mathrm{C}$, and resultant pellets lysed in SDS buffer (2\% SDS, 10\% glycerol, $60 \mathrm{mM}$ Tris-CL, $\mathrm{pH}$ 6.8, and 5\% dithiothreitol). HEK293 cells and primary macrophages were cultured and transfected with LRRK2 plasmids as previously described [7, 18]. Urine, CSF, or serum (Supplemental) samples were quickthawed in a shaking $42{ }^{\circ} \mathrm{C}$ water bath and placed on ice after thawing. Samples were centrifuged at $10,000 \mathrm{x} g$ at $4{ }^{\circ} \mathrm{C}$ for $30 \mathrm{~min}$, supernatant centrifuged at $100,000 \mathrm{x} \mathrm{g}$ for 1 hour at $4{ }^{\circ} \mathrm{C}$, and the resultant exosome-enriched pellet was washed in $1 \mathrm{~mL}$ saline and centrifuged at $100,000 \times \mathrm{g}$ for one hour at $4{ }^{\circ} \mathrm{C}$. Randomly selected exosome-enriched pellets were analyzed via singleparticle tracking Nanosight analysis. Representative vesicle size and concentration traces were recorded over five runs (60 sec per run) each and analyzed with NTA software (Nanosight).

\section{Quantification of pS1292-LRRK2 in recombinant protein standards by LC-MS}

Full-length recombinant Flag-G2019S-LRRK2 protein was purchased from Invitrogen and purity was assessed by Coomassie-stain SDS-PAGE. Concentrations of LRRK2 protein were assessed by BCA assay (Pierce). LRRK2 protein $(100 \mathrm{nM})$ was incubated with $200 \mu \mathrm{M}$ ATP in buffer containing $150 \mathrm{mM} \mathrm{NaCl}, 50 \mathrm{mM}$ Tris$\mathrm{HCl}$, and $10 \mathrm{mM} \mathrm{MgCl} 2$ at $37{ }^{\circ} \mathrm{C}$ for up to $60 \mathrm{~min}$. LRRK2 protein standards were digested with endoproteinase Glu-C (Roche) in $50 \mathrm{mM}$ Tris- $\mathrm{HCl}$ (pH7.0) at 37 ${ }^{0} \mathrm{C}$ overnight, and heavy isotope labeled peptides MGK^LSKIWDLPLDE and MGK^L(pS)KIWDLPLDE (New England Peptide, $\mathrm{K}^{\wedge}:{ }^{13} \mathrm{C}_{6}^{15} \mathrm{~N}_{2}$-lysine) were added to samples that were injected into a $300 \mu \mathrm{m} \times 5 \mathrm{~mm}$
Thermo $\mu$-Precolumn (C18 Pepmap 100, $5 \mu \mathrm{m}, 100 \AA$ pore). After washing with $0.1 \%$ formic acid and $2 \%$ acetonitrile in water at $20 \mu \mathrm{L}$ per min, $35{ }^{\circ} \mathrm{C}$, for 4.5 min, samples were separated on a $75 \mu \mathrm{m} \times 150 \mathrm{~mm}$ Thermo EASY-Spray column (C18 Pepmap, $3 \mu \mathrm{m}$ particle, $100 \AA$ pore) using a Thermo Ultimate 3000 RSLCnano LC system. Mobile phase A consisted of $0.1 \%$ formic acid in water and mobile phase B consisted of $0.1 \%$ formic acid in acetonitrile. The peptides were eluted using a linear gradient from $2 \%$ to $90 \%$ mobile phase $\mathrm{B}$ at $400 \mathrm{~nL}$ per min, $40{ }^{\circ} \mathrm{C}$, over $15.5 \mathrm{~min}$. The phospho and non-phospho S1292-containing peptides and their internal standards were detected with a Thermo Q Exactive plus Orbitrap mass spectrometer running PRM (parallel reaction monitoring). The electrospray voltage, capillary temperature, and S-lens RF were $2.0 \mathrm{kV}, 300{ }^{\circ} \mathrm{C}$, and $50 \mathrm{~V}$, respectively. The b12 and b13 fragments $(\mathrm{m} / \mathrm{z}$ values as indicated below) of the peptides were summed to generate ion chromatograms, and ratios between the peak areas of the light and heavy peptide were used for quantifying the phospho and non-phospho S1292 peptides from recombinant LRRK2 protein to calculate the degrees of phosphorylation. Detection limit for the unphosphorylated peptide (MGKLSKIWDLPLDE) was estimated to be approximately $100 \mathrm{pg} \mathrm{mL}^{-1}$ while it was approximately $300 \mathrm{pg} \mathrm{mL}^{-1}$ for the phosphorylated peptide (MGKLPSKIWDLPLDE).

\begin{tabular}{|c|c|c|c|c|}
\hline Detection peptide & type & $\begin{array}{l}\text { Precursor } \\
\text { ion }(\mathrm{m} / \mathrm{z})\end{array}$ & $\begin{array}{l}\text { Product ion } \\
(\mathrm{m} / \mathrm{z})\end{array}$ & $\mathrm{NCE}(\mathrm{V})$ \\
\hline MGKLSKIWDLPLDE & $\begin{array}{l}\text { Surrogate } \\
\text { peptide }\end{array}$ & 548.96 & $\begin{array}{l}691.8969 \text { (b12) } \\
749.4103 \text { (b13) }\end{array}$ & 15 \\
\hline MGKLSKIWDLPLDE & $\begin{array}{l}\text { Internal } \\
\text { standard }\end{array}$ & 551.63 & $\begin{array}{l}695.9040 \text { (b12) } \\
753.4174 \text { (b13) }\end{array}$ & 15 \\
\hline MGKL(pS)KIWDLPLDE & $\begin{array}{l}\text { Surrogate } \\
\text { peptide }\end{array}$ & 575.62 & $\begin{array}{l}731.8800 \text { (b12) } \\
789.3935 \text { (b13) }\end{array}$ & 14 \\
\hline MGKL(pS)KIWDLPLDE & $\begin{array}{l}\text { Internal } \\
\text { standard }\end{array}$ & 578.29 & $\begin{array}{l}735.8871 \text { (b12) } \\
793.4006 \text { (b13) }\end{array}$ & 14 \\
\hline
\end{tabular}


Table 3 Clinical data and demographics corresponding to subjects who donated both CSF and urine in the same visit

\begin{tabular}{|c|c|c|c|c|c|}
\hline & LRRK2+/PD- & LRRK2+/PD+ & LRRK2-/PD+ & LRRK2-/PD- & $p$ value $^{a}$ \\
\hline Gender M(F) & $11(15)$ & $3(12)$ & $5(6)$ & $0(3)$ & ns \\
\hline Age & $62.5(56-67.25)$ & $58(53-69)$ & $57(50-64)$ & $73(60-75)$ & ns \\
\hline Age at onset & NA & 55 (47-65) & $57(50-60)$ & NA & ns \\
\hline LEDD & NA & $600(375-662.5)$ & $400(300-600)$ & NA & ns \\
\hline UPDRS_III & $4.5(0-6)$ & $18(15-20)$ & $18(16-22.5)$ & $0(0-0)$ & $<0.0001$ \\
\hline MoCA & $26(26-28)$ & $26(25-27)$ & $27(26-28)$ & $26(<26-30)$ & 0.01 \\
\hline H\&Y & $0(0-0)$ & $2(2-2.5)$ & $2(2-2)$ & $0(0-0)$ & $<0.0001$ \\
\hline
\end{tabular}

LEDD is L-dopa equivalent daily dosage, UPDRS is Unified Parkinson's Disease Rating Scale, MoCA is Montreal Cognitive Assessment, H\&Y is Hoehn \& Yahr scale, NA is not applicable; Values reported are group median (interquartile range). ${ }^{a}$ Determined by comparing LRRK2+/PD- to LRRK2+/PD+ using the Dunn post hoc test following Kruskal-Wallis test of all groups. $n s$ is not significant as determined by Pearson chi square (gender), ANOVA (age at test), $t$ test (age at onset), Mann-Whitney test (LEDD) and Kruskal-Wallis test (H\&Y, UPDRS III)

\section{Quantification of proteins by immunoblot}

To measure the amount of total LRRK2 and pS1292LRRK2 in clinical samples, a pool was first created by combining $1 / 10(\mathrm{w} / \mathrm{v})$ of all samples from the experiment used in each analytical run. The amount of pS1292-LRRK2 and total LRRK2 protein in the pool sample was determined by comparing the pool together with recombinant protein analyzed by mass spectrometry to determine absolute values of total LRRK2 and pS1292-LRRK2 via immunoblot method (see Additional file 1: Figure S1 and S2). The amount of pS1292-LRRK2 and total LRRK2 protein in the HEK293 cell and exosome samples was determined similarly, with WTLRRK2, pathogenic mutations G2019S, R1441G and double LRRK2 mutation R1441C/G2019S samples that covers the entire signal range of LRRK2 and comparing with recombinant protein previously analyzed by mass spectrometry. Samples were electrophoresed on $4-20 \%$ TGX gradient gels (BioRad) and transferred onto PVDF membranes (Immobilon-FL, Millipore). Membranes were blocked using 5\% non-fat milk in TBST and cut in half for analysis of LRRK2 in the top half and exosomal housekeeper proteins Tsg101 or flotillin-1 in the bottom half. Digital signal intensities were recorded with the ChemiDoc Touch platform and ImageLab software (BioRad). Linearity of digital signals were confirmed across the possible dynamic range (10-600pg depending on antibody conditions, see Additional file 1: Figure S1 and S4).

The following antibodies were used: N241A/34 antiLRRK2 (Antibodies Inc), MJFF2 c41-2 anti-LRRK2 (Abcam), UDD3 anti-LRRK2 (Abcam), UDD2-10 anti-pS935 LRRK2 (Abcam), MJFR-19-7-8 anti-pS1292-LRRK2, UDD3 antiLRRK2 (Abcam), D2V7J anti-flotillin-1 (Cell Signaling), and polyclonal antibodies to phospho-threonine (P-Thr-Poly 9381, Cell Signaling) and Tsg101 (ab30871, Abcam).

\section{Phosphatase treatment of membranes}

For some immunoblots, after transfer of protein was completed, membranes were blocked with 3\% BSA
(Jackson ImmunoResearch) in TBS-T and treated with or without 250 units $\mathrm{mL}^{-1}$ calf intestinal alkaline phosphatase (CIP, New England BioLabs) in CutSmart buffer (New England BioLabs) for 2 hours at $37{ }^{\circ} \mathrm{C}$. Quantification of proteins on the membranes then proceeded as described above.

\section{Statistics and figures}

All samples were assayed and quantified in randomized order and group assignments were made after final data curation. One-way ANOVAs with Tukey post hoc test, or unpaired t-test, was used for parametric continuous variables to compare group means. Kruskal-Wallis oneway analysis with Dunn test for post hoc comparison, or Mann-Whitney U test, were used to compare median values of categorical and nonparametric variables. Correlation analyses were performed using Spearman Rank-Order tests to accommodate nonparametric variables. All significance levels are reported as two-tails, corrected for multiple testing, with $p<0.05$ interpreted as significant. Statistical analysis was performed using JMP Pro 10 (SAS Institute Inc), graphs built with GraphPad Prism 5.0 and arranged in Adobe Illustrator 10.

\section{Results \\ Exosome pS1292-LRRK2 levels correlate with cellular LRRK2 kinase activity}

Previously, we demonstrated that heterozygous G2019SLRRK2 mutations increased the ratio of pS1292-LRRK2 to total LRRK2 protein $\sim 4$-fold in urinary exosomes of G2019S carriers as compared to non-carriers [6]. These and other measurements from model systems have not revealed the proportion of LRRK2 protein phosphorylated at pS1292, as only relative ratios of signal have been presented in past studies $[6,8,14,23]$. Further, it is not known how different $L R R K 2$ mutations and different cellular and exosome sources may differ in the amount of pS1292-LRRK2 with respect to other LRRK2 kinase substrates like Rab10 [26]. In measuring pS1292-LRRK2 from in vitro LRRK2 kinase reactions, we observed a 
low-starting level of pS1292-LRRK2 in recombinant G2019S-LRRK2 protein that increased over time, as expected (Fig. 1a). In contrast, the constitutive phosphorylation site pS935 that is not a LRRK2 autophosphorylation site did not change over time. Other threonine LRRK2 autophosphorylation sites have been described in the LRRK2 ROC GTPase domain [10, 12, 31]. Cumulatively, these increased in abundance over time like pS1292 as revealed by pan-phospho-threonine detection (Fig. 1a). Thus, in vitro, pS1292-LRRK2 levels correlate closely with overall autophosphorylation. Using a novel mass spectrometry approach to quantify pS1292LRRK2 over time in recombinant protein, we could determine that $45 \%$ of the LRRK2 protein was pS1292LRRK2 after one-hour (Fig. 1b). Cellular levels of immunoprecipitated pS1292-LRRK2 (before kinase assays) demonstrated only $\sim 2 \%$ of total LRRK2 is pS1292LRRK2 (Fig. 1a, b). To verify the specificity of the
pS1292-LRRK2 antibody for a phosphorylated peptide, we introduced a Ser1292Ala mutation into the G2019SLRRK2 backbone and found that the Ser1292Ala mutation blocks pS1292-LRRK2 antibody binding (Fig. 1c).

We next compared the effect of pathogenic LRRK2 mutations on pS1292-LRRK2 levels in both cell extracts from transfected HEK293 cells as well as extracellular exosomes purified from their cell culture media. As expected, all the pathogenic LRRK2 mutations increased cellular levels of pS1292-LRRK2 (Fig. 1d), with the highest levels induced by a double-mutation R1441C / G2019S, also known to enhance neurotoxicity in primary neurons [23]. pS1292-LRRK2 levels were similar between WT-LRRK2, the prevalent Far-East Asian G2385R-LRRK2 risk variant, and the non-pathogenic I2012A-LRRK2 variant. Through comparison to recombinant pS1292-LRRK2 standards defined by mass spectrometry (and in linear range, see Additional file 1:

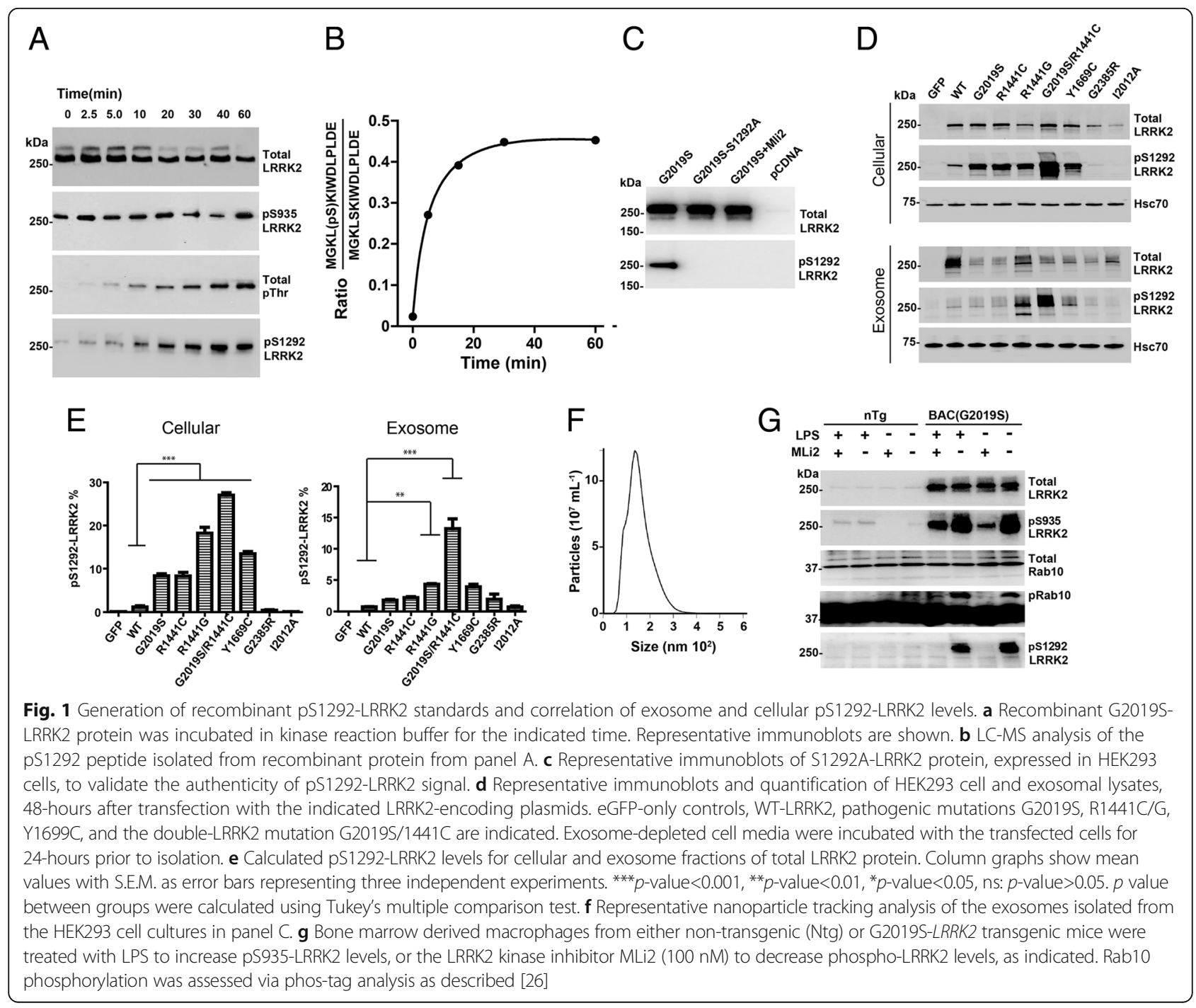


Figure S2), we deduced the percent of pS1292-LRRK2 in cells in the double-LRRK2 mutant as $\sim 27 \%$ of available LRRK2 protein (Fig. 1d,e).

Exosome protein levels do not usually reflect the levels of proteins and their post-translational modifications found in cell cytosols due to the mechanisms that select for exosome cargo and transport in the endolysosomal system [28]. However, exosomes from the transfected HEK293-derived exosomes revealed similar pS1292LRRK2 levels as compared to the parental cellular lysates (Fig. 1d, e). The effects of the double-mutation G2019S/ R1441C appeared slightly exaggerated compared to other mutations, and in general, pS1292-LRRK2 levels were slightly lower on average (Fig. 1e). Analysis of the exosome pellets from these cells via single-particle nanotracking revealed a typical microvesicle population that had an average size of $134 \mathrm{~nm}$ (Fig. 1f). Thus, in these cells, exosomes reported pS1292-LRRK2 levels that were consistent with cellular levels that were increased by pathogenic $L R R K 2$ mutations.

In humans, $L R R K 2$ expression is highest in circulating leukocytes in the blood according to RNAseq tissue expression profiles [32]. Leukocytes like macrophages and monocytes may secrete exosomes to control some aspects of immune reactions [15]. To measure endogenous LRRK2 protein in macrophages in culture, we isolated exosomes and cellular lysates from wild-type or G2019S-LRRK2 expressing macrophages. In cellular lysates, we could not reliably detect any pS1292-LRRK2 in wild-type macrophages, whereas pS1292-LRRK2 levels were robust with G2019S-LRRK2 expression (Fig. 1g). pS1292-LRRK2 signal, as well as pRab10 signal (a trans LRRK2 kinase substrate), was ablated with one-hour treatment of a LRRK2 kinase inhibitor. However, we were unable to detect pS1292-LRRK2 or total LRRK2 protein in exosomes isolated from primary macrophages, irrespective of LPS stimulation. These results suggest that leukocytes like macrophages that express high LRRK2 levels may not be an important source for LRRK2 protein in exosomes.

\section{Brain-derived exosomes have elevated pS1292-LRRK2 levels}

Previously we detected total LRRK2 protein in exosomeenriched fractions purified from post-mortem remnant CSF [7]. To determine whether we can detect pS1292LRRK2 in biobanked clinical CSF samples verified to have low or no detectable hemoglobin, we analyzed three samples from neurologically normal controls provided by the BioFIND repository. Guided by Nanosight analysis of microvesicle fractions, we applied a modified differential ultracentrifugation strategy to isolate an exosome fraction that harbors vesicles with an average size of $\sim 125 \mathrm{~nm}$ (Fig. 2a, b). These exosomes were similar in characteristics to those isolated from HEK293 cells. Using a reference pS1292-LRRK2 protein, we detected appreciable signal for total LRRK2 and pS1292-LRRK2, but only in the exosome-enriched fraction and not in supernatants or low speed $(10,000 \times \mathrm{x}$ ) pellets (Fig. 2c). The signal elicited by the pS1292-LRRK2 antibody in the human CSF exosome pellet was destroyed by brief treatments of the membranes with calf intestinal alkaline phosphatase (CIP), indicating that the cross-reactive band is a phospho-protein and of the exact molecular weight as our full-length recombinant protein standard ( $280 \mathrm{kDa}$, Fig. 2d). As opposed to HEK293, macrophage, and urinary exosome lysates, a significant immunoglobulin signal due to the species secondary antibody is apparent at $\sim 90 \mathrm{kDa}$, as well as a lowintensity band (compared to pS1292-LRRK2) at $\sim 70$ $\mathrm{kDa}$. In these CSF specimens, hemoglobin levels were below our ELISA detection limit $\left(<2 \mathrm{pg} \mathrm{mL}^{-1}\right)$, suggesting LRRK2 protein in the CSF is probably not from blood product contamination as our lower detection limit for pS1292-LRRK2 is higher than $2 \mathrm{pg} \mathrm{mL}^{-1}$.

In a recent past study, a 'pooled' exosome fraction was created that is composed of urinary exosome lysates from $\sim 160$ subjects with and without PD [8]. Using our analytical process described here, we can estimate $\sim 5 \%$ of total LRRK2 in this pools sample is pS1292-LRRK2 (Fig. 2e). In comparing the BioFIND CSF exosome lysates, total LRRK2 protein was lower but pS1292-LRRK2 was much higher and calculated at $\sim 80 \%$ on average (Fig. 2e). This level of pS1292-LRRK2 was higher than that we recorded from urinary exosomes from LRRK2 mutation carriers, higher than that we could achieve by in vitro kinase assays with recombinant protein, and higher than from HEK293 exosomes transfected with our double-LRRK2 mutation construct. Cryo-electron microscopy analysis of the CSF exosome pellets revealed a similar microvesicle constituency as our past observations with urinary exosomes [7] and single-particle tracking measurements here (Fig. 2f).

\section{Elevated pS1292-LRRK2 levels in Norwegian LRRK2 mutation carriers}

Previously, we found an elevated ratio of pS1292-LRRK2 normalized to total LRRK2 protein and the control exosome protein Tsg101 in urinary exosomes isolated from male G2019S-LRRK2 mutation carriers from the MJFF LRRK2 Cohort [6]. Here, we sought to determine whether a similar relationship exists in a Norwegian cohort of LRRK2 mutation carriers, with and without PD, and extended the analysis to include females as well as an absolute quantification of pS1292-LRRK2. The cohort we analyzed consisted of 132 subjects that donated urine (Table 1), 82 subjects that agreed to donate CSF (Table 2), 


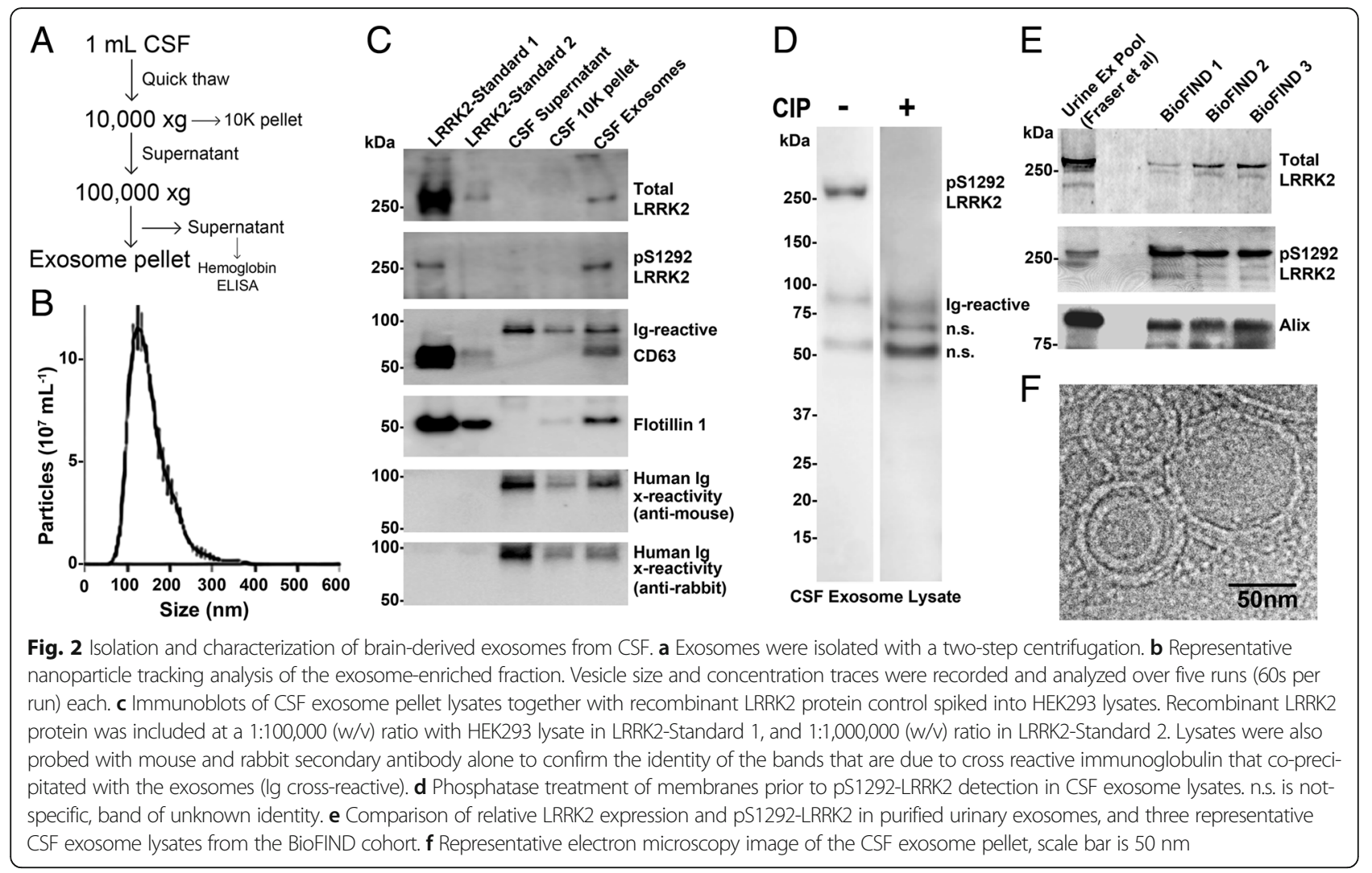

and 55 subjects that donated both CSF and urine in the same clinic visit (Table 3).

Measurements of pS1292-LRRK2 levels in urinary exosomes, normalized to the abundance of the control exosome protein Tsg101, revealed elevated pS1292-LRRK2 in G2019S-LRRK2 mutation carriers compared to noncarriers ( 4.8 fold on average, $\mathrm{p}<0.0001$, Fig. 3a1). In breaking groups according to sex, male G2019S-LRRK2 mutation carriers with PD had higher pS1292-LRRK2/ Tsg101 levels than in carriers without PD ( $~ 8.9$ fold versus $\sim 3.8$ fold, $\mathrm{p}=0.04)$. However, in the female group, this trend was reversed ( 3.6 fold versus $\sim 5.4$ fold, $\mathrm{p}=0.012$, Fig. 3a3). Thus, the female mutation carriers with PD had similar levels of pS1292-LRRK2 as the male carriers, but the age-matched female LRRK2 mutation carriers without PD also had high levels. We did not detect significant correlations between pS1292-LRRK2/ Tsg101 levels and any urine characteristic we measured in the samples (leukocyte count, pH, glucose, total protein, red-blood cells, and specific gravity), or any demographic or clinical data (Spearman $\mathrm{R}$ values, all $p$ values $>0.1$, Table 1).

In CSF exosome isolations from the cohort, we could not reliably detect the exosome protein Tsg101 whereas we could reliably measure the exosomal housekeeping protein flotillin-1 in the samples. Analysis of CSF for pS1292-LRRK2 levels, as normalized to the abundance of flotillin-1, revealed similar amounts in the groups irrespective of $\mathrm{PD}$ diagnosis, $L R R K 2$ mutation status, or sex (Fig. 3b). In 60 of 81 CSF samples analyzed, hemoglobin levels were below the limits of reliable detection using our ELISA platform $\left(<2 \mathrm{pg} \mathrm{mL}^{-1}\right)$. Of the remaining samples with measured hemoglobin, there was no correlation between pS1292-LRRK2 / flotillin-1 or other protein measurements (Spearman $\mathrm{R}<0.1$, all $p>0.4)$. Two CSF specimens had particularly high hemoglobin levels $>200 \mathrm{pg} \mathrm{mL}^{-1}$, but these samples had average pS1292-LRRK2, total LRRK2, and flotillin-1 protein levels. These results demonstrate that we could readily measure pS1292-LRRK2 in CSF exosome fractions in a large biobanked series, although levels were not different in LRRK2 mutation carriers despite the robust differences we could observe in urine collected in the same clinic visit in the same subjects.

We hypothesized that the reason pS1292-LRRK2/flotillin-1 levels in CSF could not identify LRRK2 mutation carriers was related to the very high proportion of pS1292-LRRK2 in CSF and thus possible ceiling effects inherent to limited substrate (i.e., total LRRK2 protein). We therefore defined the percent of LRRK2 phosphorylated at the 1292 residue in each urine and CSF exosome sample. As expected, we found an elevated proportion of pS1292-LRRK2 in urinary exosomes in LRRK2 mutation carriers compared to non-carriers $(7.3 \%$ of total LRRK2 


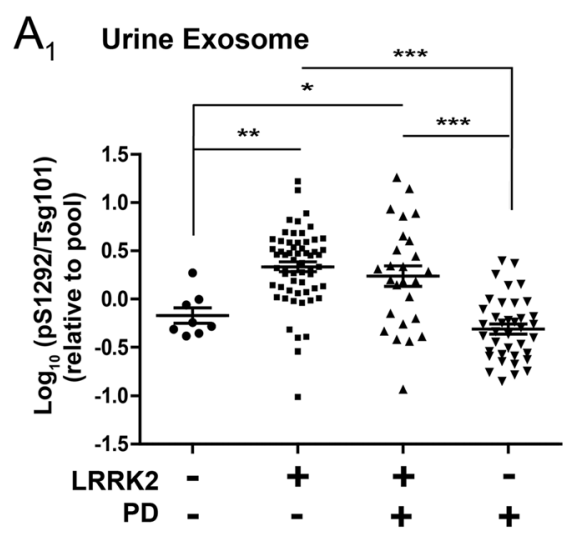

\section{$B_{1}$ CSF Exosome}
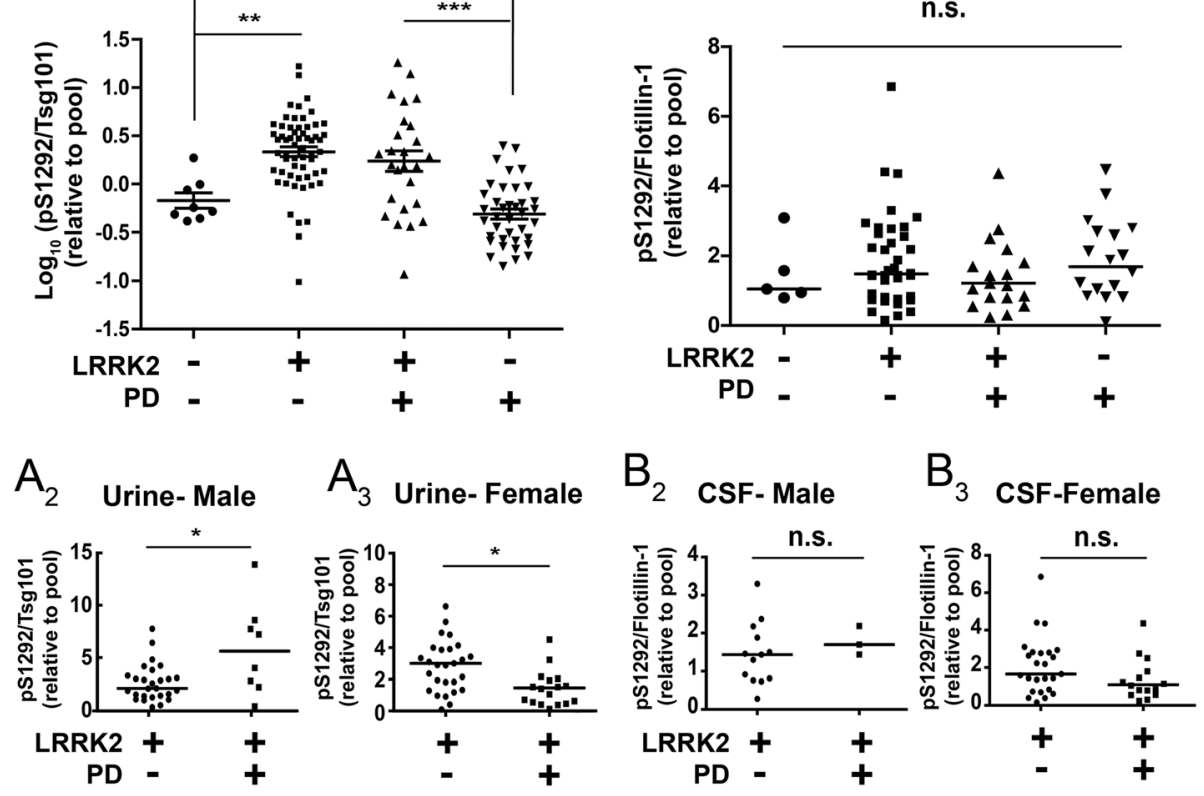

$\mathrm{B}_{3}$ csF-Female

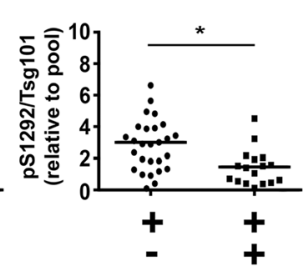

Fig. 3 Quantification of exosomal pS1292-LRRK2 in urine and CSF (a) Scatter plots showing pS1292-LRRK2 expression levels normalized to TSG101 expression, relative to the pool (all samples, $\mathrm{N}=132$ ). Bars depict mean values with error bars showing S.E.M. Quantifications were based on the average value of three independent immunoblot runs. $\mathbf{A}_{\mathbf{2}, \mathbf{3}}$ The LRRK2 mutation carrier groups (PD+/-) are broken further according to sex as indicated. Bars show median values. b Scatter plots showing relative pS1292-LRRK2 expression level normalized to flotillin-1 expression, relative to the pool (all samples, $\mathrm{N}=81$ ). The LRRK2 mutation carrier groups ( $\mathrm{PD}+/-)$ are broken further according to sex as indicated $\left(\mathbf{B}_{\mathbf{2}, \mathbf{3}}\right)$. Bars show median values. ${ }^{* * *} p$-value $<0.001,{ }^{* *} p$-value $<0.01,{ }^{*} p$-value $<0.05$, ns: $p$-value $>0.05$. $p$ value between groups were calculated using Tukey's multiple comparison test (figure $\mathbf{a}_{1}$ and $\mathbf{b}_{1}$ ) and Mann-Whitney test (figure $\mathbf{a}_{2-3}$ and $\mathbf{b}_{2-3}$ )

protein in mutation carriers versus $4.2 \%$ in non-carriers, $p<0.0001$, Fig. 4a). Breaking these groups according to sex, consistent with pS1292-LRRK2/Tsg101 measurements, male carriers with PD had significantly higher phosphorylation at the 1292 residue than carriers without PD (11.5\% versus $6.4 \%$, respectively, $p=0.0239$ Fig. 4a2). Samples from females with a LRRK2 mutation and PD again showed a slightly lower percent pS1292LRRK2 than mutation carriers without PD, although the difference was not significant $(6.5 \%$ versus $9.4 \%$, respectively, $\mathrm{p}=0.26$, Fig. 4a3).

These analyses in CSF pS1292-LRRK2 levels revealed an unexpected ceiling effect in many samples (Fig. 4). Of the 22 CSF samples from non-LRRK2 mutation carriers,
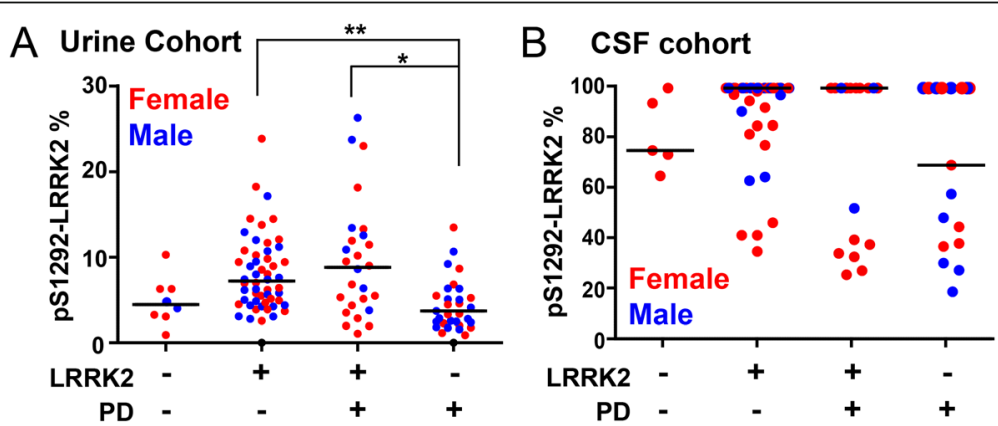

Fig. 4 pS1292-LRRK2 levels in urine and saturation in CSF Absolute levels of pS1292-LRRK2 were normalized to total LRRK2 protein to derive a ratio of the percent of total LRRK2 that is autophosphorylated. a Scatter plots showing percent pS1292-LRRK2 from urinary exosomes ( $N=132$, blue: male, red: female). b Scatter plots showing percent pS1292-LRRK2 from CSF exosomes (N=81, blue: male, red: female). Values above 100\% phosphorylation were considered within technical error of detection of full saturation and plotted as $100 \%$ for visualization purposes only and not for statistical analysis. Unadjusted absolute values of pS1292-LRRK2 are given in Fig. 5. Bars show median values. ${ }^{* * *} p$-value<0.001,

${ }^{* *} p$-value $<0.01,{ }^{*} p$-value<0.05, ns: $p$-value $>0.05$. $p$ value between groups were calculated using Dunn's multiple comparison test (a and $\mathbf{b}$ ) 
$50 \%$ (11 of 22) demonstrated $>95 \%$ phosphorylation, whereas in LRRK2 mutation carriers, 38 of 57 CSF (67\%) samples demonstrated $>95 \%$ phosphorylation. Stratification of samples that have near-saturated pS1292-LRRK2 (>95\%) from those with lower levels $(<95 \%)$ also did not successfully separate $L R R K 2$ mutation carriers from noncarriers (chi-square 1.872, $\mathrm{p}=0.17$ ) or reveal a correlation with clinical scales for PD severity.

\section{pS1292-LRRK2 correlates with total LRRK2 protein, but urine levels do not predict CSF levels.}

Evidence both in vitro in primary cultured cells, as well as in rodents and monkeys treated with LRRK2 kinase inhibitors, suggests that LRRK2 kinase activity may enhance protein stability and decrease LRRK2 protein turnover [9, 23, 26, 32]. In considering the absolute amounts of protein on a volumetric basis with no normalization to other exosome proteins, in the average $\mathrm{mL}$ of CSF, there is $\sim 120 \mathrm{pg}$ of LRRK2 harbored in exosome fractions, with only a slightly lower amount of pS1292-LRRK2, whereas in urine there is much less pS1292-LRRK2 protein but similar total LRRK2. In both urine and CSF, a strong positive correlation exists between pS1292-LRRK2 and total LRRK2 (Spearman's rho 0.76 and 0.38 , respectively, $\mathrm{p}<0.0001$ for both), giving further confidence that the pS1292-LRRK2 signal, always measured on different immunoblots than total LRRK2, is authentic in both urine and CSF.

While more LRRK2 protein positively predicts more pS1292-LRRK2 protein in both CSF and urine exosomes, we wondered whether more exosomes in general in the biofluids predicts more LRRK2 protein that we can measure. In urine, there was a positive correlation between LRRK2 protein concentration and Tsg101 levels (Spearman's rho 0.52, $\mathrm{p}<0.0001$ ), showing the Tsg101 may be in the same exosome population as LRRK2, consistent with previous observations [7]. In addition, there is an overall increase in LRRK2 protein in males compared to females (2.61 in males versus 1.64 in females, $\mathrm{p}$ $=0.003)$, consistent with recent observations in a cohort from Birmingham, Alabama [8]. In contrast, in CSF exosomes, where Tsg101 protein was not reliably detected, we found that flotillin-1 poorly correlated with LRRK2 protein levels (Fig. 5). These results suggest that the majority of the pool of flotillin-1 positive exosomes may not be LRRK2 positive. Further, levels of LRRK2 normalized to Tsg101 in urine and levels of LRRK2 normalized to flotiiln-1 in CSF did not correlate with one another in subjects from our cohort (Fig. 5c, d). Irrespective of other exosomal proteins, absolute levels of both total LRRK2 protein as well as pS1292-LRRK2 protein in CSF and urine exosome fractions also did not correlate (Fig. 5e, f). These results suggest that the parental cells shedding LRRK2-positive exosomes within an individual have differential regulation of LRRK2 expression and regulation of autophosphorylation with respect to LRRK2 mutation status and PD diagnosis.

\section{Discussion}

Our results include three key observations. First, we provide additional support for pS1292-LRRK2 in exosomes as a surrogate for LRRK2 kinase activity, with the caveat that not all LRRK2-expressing cells like macrophages may contribute LRRK2 protein equally to the exosome pool. Second, we can demonstrate in a novel Norwegian cross-sectional cohort that pS1292-LRRK2 levels are elevated in urinary exosomes from G2019SLRRK2 mutation carriers compared to non-carriers, in both males and females. These results support data from model systems that suggest kinase activating effects of the prevalent G2019S-LRRK2 mutation. According to our cohort, different than in males, in females there is no predictive value of exosome pS1292-LRRK2 levels in PD diagnosis, potentially owing to the overall lower LRRK2 levels in females versus males and overall positive correlation of pS1292-LRRK2 to total LRRK2 protein in all subjects [8]. Third, we provide evidence that LRRK2 autophosphorylation at S1292 may be much higher in brain-derived exosomes compared to exosomes purified from urine and other cellular sources. Although an important limitation of the study is that lack of male healthy controls that donated CSF, the high and often saturated pS1292-LRRK2 appears to be common to both male and female subjects that did donate CSF. Since cellular and exosome levels of pS1292-LRRK2 correlate, these results may show that LRRK2 activity is much higher in brain cells that shed exosomes into CSF than in the peripheral cells that contribute to urinary exosome pools. Overall these results provide some of the first insights into the putative pathological pS1292LRRK2 species in the brain and periphery in a clinical population.

Despite the link between LRRK2 and PD that has been known for over twelve years, and the implication of LRRK2 kinase activity in PD susceptibility, few studies have yet measured LRRK2-kinase dependent phosphorylation in clinical samples. This is because LRRK2 kinase substrates have been very difficult to identify and tend to be poorly expressed. In our transfected cells, and in primary macrophages that express very high LRRK2 protein, the amount of autophosphorylated LRRK2 is much lower than in our clinical samples. We speculate that part of the difficulty in finding LRRK2 substrates can be explained by low or no kinase-active associated with LRRK2 protein when expressed in some model systems. So far, two LRRK2 kinase substrates have been identified that increase in abundance with pathogenic LRRK2 mutations, decrease in abundance with LRRK2 kinase 

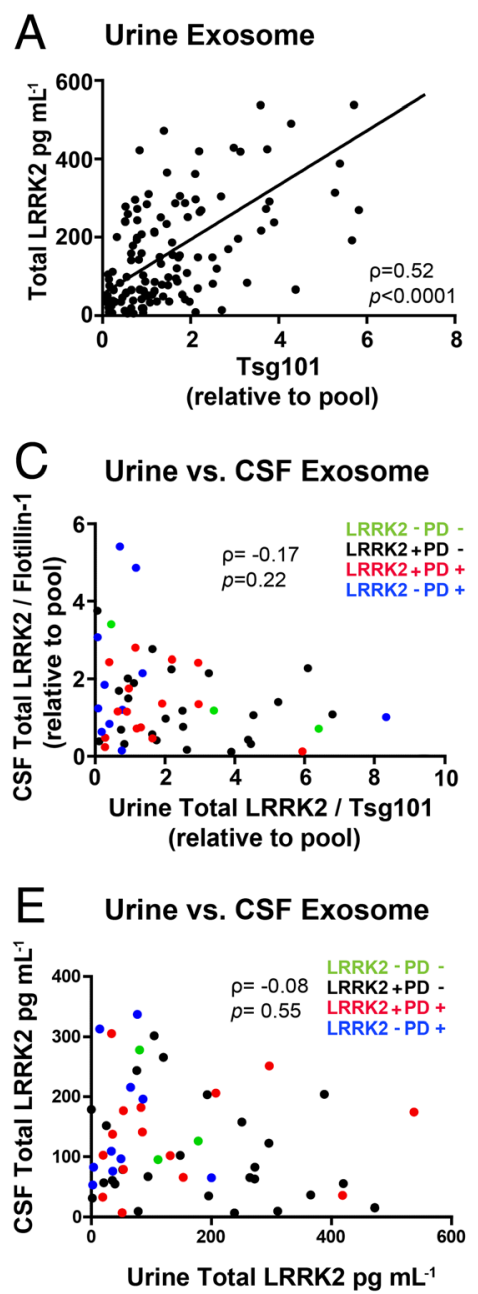

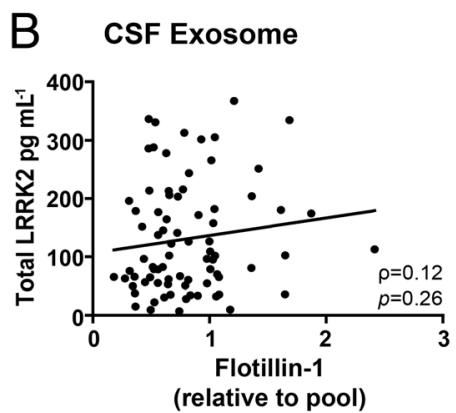

D Urine vs. CSF Exosome

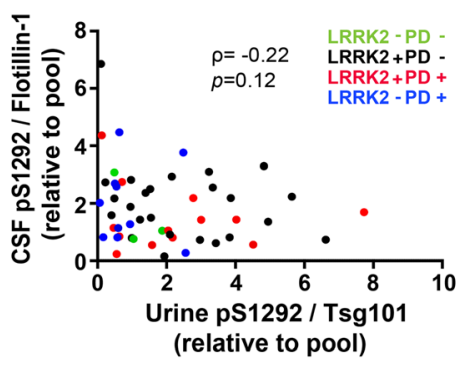

F Urine vs. CSF Exosome

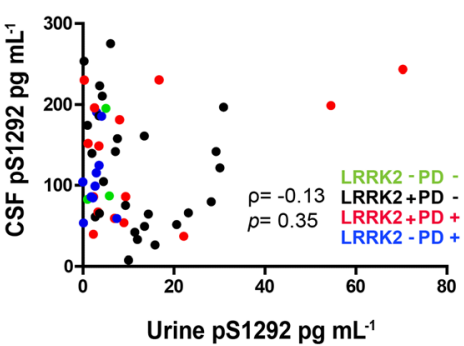

Fig. 5 Comparing urine and CSF exosomal LRRK2-expression and phosphorylation profiles. a Correlation of total LRRK2 and TSG101 in urine $(\mathrm{N}=132)$, and (b) total LRRK2 and flotillin-1 in CSF ( $N=81)$. c Correlation of total exosomal LRRK2 protein in CSF and urine, and (d) pS1292-LRRK2 in CSF and urine. Without adjusting for housekeeping exosome protein concentrations, correlation for (e) total LRRK2 protein and (f) pS1292-LRRK2 protein in CSF and urine. Samples were matched to the same clinic visit. ( $N=56$, green: LRRK2- PD-, black: LRRK2+ PD-, red: LRRK2+ PD+, blue; LRRK2- PD+). Spearman's rank correlation coefficients are shown with corresponding $p$ values

inhibition, and serve as substrates in vitro: LRRK2 protein itself via pS1292 [23] in cis-autophosphorylation, and other small-GTPase Rabs that share similarity with the LRRK2 GTPase domain that include Rab10 and Rab8 [26]. Unfortunately, we were unable to detect robust levels of Rab10 or Rab8 in exosome fractions from either urine or CSF. However, our results show that pS1292-LRRK2 can be detected and measured in both urine and CSF exosomes.

Exosome-derived LRRK2 and pS1292-LRRK2 have been analyzed using traditional immunoblotting techniques for the current study. It must be noted that measurements of exosome-derived LRRK2 has not been replicated in orthogonal assays like ELISA, singleparticle detection, or the LC-MS assay used here to measure recombinant pS1292-LRRK2. Caution should be used in data interpretation until multiple types of scalable assays demonstrate consistent congruency in measures related to LRRK2 in biofluids. In the meantime, well-characterized recombinant protein standards that help define absolute concentrations have demonstrated utility to interpretation.

There are several lines of evidence that the pS1292LRRK2 signal we measured here is authentic: First, the pS1292-LRRK2 signal disappears with LRRK2 kinase inhibitors or mutation of Ser1292 to alanine, and is increased in abundance with pathogenic LRRK2 mutations in both model systems and urinary exosomes. Second, the pS1292-LRRK2 signal, as in CSF exosomes, is dependent on phosphorylation, since phosphatase treatment of membranes ablates signal. Third, the pS1292LRRK2 signal is strongly correlated with total LRRK2 
signal, and the LRRK2 antibodies used here are raised against epitopes distant from the pS1292-LRRK2 epitope. While only one antibody epitope can be used for pS1292-LRRK2 detection, several high-quality total LRRK2 monoclonal antibodies are available and the signal these generate are perfectly correlated in the subject exosome lysates, indicating they detect the same protein (Additional file 1: Figure S3).

The rationale for LRRK2 protein detection in exosome fractions began with our initial microscopy studies where we and others could observe a small proportion of LRRK2 protein associated with intra-luminal vesicles in multi-vesicular bodies [3, 7]. First in transfected HEK293 cells, we could demonstrate that LRRK2 protein can be measured in extracellular fractions enriched in exosomes, and the pS1292-LRRK2 levels are correlated with the cellular levels. As compared to measurements of other proteins in the extracellular milieu, exosomes provide an ideal source for protein since the robust protective lipid-bilayer shields the exosome proteins from extracellular enzymes that might include phosphatases and proteases [28]. Further, the exosome proteome with hundreds of proteins is extremely reduced in complexity compared to the complexity of tissue proteomes with thousands of proteins [22, 27].

While exosomes can be isolated from all known biological fluids, we and others have not $a$ priori been able to predict which proteins and modifications can be measured in exosomes based on the cellular constituency of the parental sources. For example, we have been so-far unable to reliably detect LRRK2 expression in exosomes isolated from serum (Additional file 1: Figure S5), despite the abundance of LRRK2 expression in circulating leukocytes. This result, in combination with measures of hemoglobin in CSF, suggest that LRRK2 protein in CSF we measured is unlikely to be related to blood contamination. There is yet little information on the origins of the diversity of microvesicle subpopulations in clinical samples like CSF and urine. Thus, the types of cells in the body that contribute LRRK2-positive exosomes in the periphery in urine and in the brain in CSF are unknown. Furthermore, we are unable to assess the degree of variability, regarding to total exosome number, size, and distribution between individuals and groups because we were unable to perform nanoparticle tracking in every sample due to limitations in sample volumes available. Future studies will be critical in establishing the normal biology of different subsets of exosomes and microvesicles. In this study and our past studies, pS1292-LRRK2 levels in urinary exosomes have been robust in separating LRRK2 mutation carriers from non-carriers $[6,8]$. Given the striking differences in the amount of LRRK2 autophosphorylation that occurs in the brain versus peripheral exosomes,
LRRK2 kinase activity does not appear hard-coded intrinsically within the protein as has been suspected, but regulated by an external network of factors. This observation appears to hold true in mice as well, since LRRK2 protein isolated from the lung has much less kinase activity than the same amount of LRRK2 isolated from the brain [16]. This may reflect a unique aspect of LRRK2 biology in the human brain that may be important for PD susceptibility.

Several direct applications and extensions based on our work here can be envisaged. First, in an independent cohort with subjects on a different ethnic background (Norwegian), in males, but not females, we could confirm the potential of pS1292-LRRK2 levels in urine as a candidate biomarker for PD susceptibility, where non-manifesting subjects tended to have lower pS1292LRRK2 levels. Notably, our sample size of male G2019S carriers with PD was half that of our first study $(\mathrm{N}=8$ here versus $\mathrm{N}=16$ in our past study) and a difference was still detected. Thus, urinary exosome pS1292LRRK2 levels may help identify male mutation carriers at the highest risk for PD onset, together with other emerging biomarkers. Future studies using highly specific, sensitive and scalable orthogonal methods of LRRK2 and phosphorylated LRRK2 quantification are critically needed for the translation of our results to clinical utility.

\section{Conclusions}

The G2019S-LRRK2 mutation upregulates LRRK2-kinase activity-dependent autophosphorylation at Ser1292 in exosomes captured from peripheral and brain-derived exosomes. LRRK2 protein in brain exosomes may be much more active than in the periphery in most subjects.

\section{Additional files}

Additional file 1: Supplemental figures. (PDF 5036 kb)

\section{Abbreviations}

COR: C-terminal of ROC; CSF: Cerebral spinal fluid; H\&Y: Hoehn \& Yahr scale; LEDD: L-dopa equivalent daily dose (LEDD); LRRK2: Leucine rich repeat kinase 2; MoCA: Montreal Cognitive Assessment; mS\&E: Schwab \& England scale; PD: Parkinson's disease; ROC: Ras-of-complex; UPDRS: Unified Parkinson's

Disease Rating Scale

\section{Acknowledgements}

The authors thank Yijan Zhang for assistance in exosome purifications.

\section{Funding}

This work was supported by the Michael J. Fox Foundation for Parkinson's disease research and the National Institutes of Health grants U01 NS097028, R01 NS064934, R21 NS097643, and the American Parkinson's Disease Association.

\section{Availability of data and materials}

The datasets used and/or analysed during the current study are available from the corresponding author on reasonable request. 


\section{Authors' contributions}

SW, OM, JA and ABW wrote the manuscript, SW, ZL, TM, and TY performed the experiments, SW, ABW, ZL analyzed the experimental data, and JA conceived the cohort and oversaw all clinical data and demographic data collection and analysis. All authors read and approved the final manuscript.

\section{Ethics approval and consent to participate}

All protocols were approved by the local Institutional Review Boards, and all participants were recruited and consented to participate in the outpatient clinic of the Department of Neurology, St. Olavs hospital, Trondheim, Norway

\section{Consent for publication}

Not applicable.

\section{Competing interests}

TY and OSM are employees of Biogen Inc. The authors declare no other competing financial or non-financial interests.

\section{Publisher's Note}

Springer Nature remains neutral with regard to jurisdictional claims in published maps and institutional affiliations.

\section{Author details}

'Department of Neurology, Center for Neurodegeneration and Experimental Therapeutics, University of Alabama at Birmingham, Birmingham, AL 35233, USA. ${ }^{2}$ Biogen, Discovery and Early Development Biomarkers, Cambridge, MA 02142, USA. ${ }^{3}$ Dept of Neurology, Dept of Neuroscience, St. Olavs Hospital, Norwegian University of Science and Technology, 7030 Trondheim, Norway.

\section{Received: 29 September 2017 Accepted: 8 November 2017}

\section{Published online: 22 November 2017}

\section{References}

1. Aasly JO, Shi M, Sossi V, Stewart T, Johansen KK, Wszolek ZK, Uitti RJ, Hasegawa K, Yokoyama T, Zabetian CP et al (2012) Cerebrospinal fluid amyloid beta and tau in LRRK2 mutation carriers. Neurology 78:55-61. doi:10.1212/WNL.0b013e31823ed101

2. Alcalay RN, Mirelman A, Saunders-Pullman R, Tang MX, Mejia Santana H, Raymond D, Roos E, Orbe-Reilly M, Gurevich T, Bar Shira A et al (2013) Parkinson. disease phenotype in Ashkenazi Jews with and without LRRK2 G2019S mutations. Mov Disord 28:1966-1971. doi:10.1002/mds.25647

3. Biskup S, Moore DJ, Celsi F, Higashi S, West AB, Andrabi SA, Kurkinen K, Yu SW, Savitt JM, Waldvogel HJ et al (2006) Localization of LRRK2 to membranous and vesicular structures in mammalian brain. Ann Neurol 60: 557-569. doi:10.1002/ana.21019

4. Cho HJ, Liu G, Jin SM, Parisiadou L, Xie C, Yu J, Sun L, Ma B, Ding J, Vancraenenbroeck $R$ et al (2013) MicroRNA-205 regulates the expression of Parkinson's disease-related leucine-rich repeat kinase 2 protein. Hum Mol Genet 22:608-620. doi:10.1093/hmg/dds470

5. Dzamko N, Gysbers AM, Bandopadhyay R, Bolliger MF, Uchino A, Zhao Y, Takao M, Wauters S, van de Berg WD, Takahashi-Fujigasaki J et al (2017) LRRK2 levels and phosphorylation in Parkinson's disease brain and cases with restricted Lewy bodies. Mov Disord 32:423-432. doi:10.1002/mds.26892

6. Fraser KB, Moehle MS, Alcalay RN, West AB, Consortium LC (2016) Urinary LRRK2 phosphorylation predicts parkinsonian phenotypes in G2019S LRRK2 carriers. Neurology 86:994-999. doi:10.1212/WNL.0000000000002436

7. Fraser KB, Moehle MS, Daher JP, Webber PJ, Williams JY, Stewart CA, Yacoubian TA, Cowell RM, Dokland T, Ye T et al (2013) LRRK2 secretion in exosomes is regulated by 14-3-3. Hum Mol Genet 22:4988-5000. doi:10.1093/hmg/ddt346

8. Fraser KB, Rawlins AB, Clark RG, Alcalay RN, Standaert DG, Liu N, Parkinson's Disease Biomarker Program C, West AB (2016) Ser(P)-1292 LRRK2 in urinary exosomes is elevated in idiopathic Parkinson's disease. Mov Disord 31:15431550. doi:10.1002/mds.26686

9. Fuji RN, Flagella M, Baca M, Baptista MA, Brodbeck J, Chan BK, Fiske BK, Honigberg L, Jubb AM, Katavolos Pet al (2015) Effect of selective LRRK2 kinase inhibition on nonhuman primate lung. Sci Transl Med 7: 273ra215 doi:10.1126/scitransImed.aaa3634

10. Gloeckner CJ, Boldt K, von Zweydorf F, Helm S, Wiesent L, Sarioglu H, Ueffing M (2010) Phosphopeptide analysis reveals two discrete clusters of phosphorylation in the N-terminus and the Roc domain of the Parkinson- disease associated protein kinase LRRK2. J Proteome Res 9:1738-1745. doi:10.1021/pr9008578

11. Greggio E, Jain S, Kingsbury A, Bandopadhyay R, Lewis P, Kaganovich A, van der Brug MP, Beilina A, Blackinton J, Thomas KJ et al (2006) Kinase activity is required for the toxic effects of mutant LRRK2/dardarin. Neurobiol Dis 23: 329-341. doi:10.1016/j.nbd.2006.04.001

12. Greggio E, Taymans JM, Zhen EY, Ryder J, Vancraenenbroeck R, Beilina A, Sun P, Deng J, Jaffe H, Baekelandt V et al (2009) The Parkinson's disease kinase LRRK2 autophosphorylates its GTPase domain at multiple sites. Biochem Biophys Res Commun 389:449-454. doi:10.1016/j.bbrc.2009.08.163

13. Healy DG, Falchi M, O'Sullivan SS, Bonifati V, Durr A, Bressman S, Brice A, Aasly J, Zabetian CP, Goldwurm S et al (2008) Phenotype, genotype, and worldwide genetic penetrance of LRRK2-associated Parkinson's disease: a case-control study. Lancet Neurol 7:583-590. doi:10.1016/S1474-4422(08)70117-0

14. Henry AG, Aghamohammadzadeh S, Samaroo H, Chen Y, Mou K, Needle E, Hirst WD (2015) Pathogenic LRRK2 mutations, through increased kinase activity, produce enlarged lysosomes with reduced degradative capacity and increase ATP13A2 expression. Hum Mol Genet 24:6013-6028. doi:10.1093/hmg/ddv314

15. Lee HD, Koo BH, Kim YH, Jeon OH, Kim DS (2012) Exosome release of ADAM15 and the functional implications of human macrophage-derived ADAM15 exosomes. FASEB J 26:3084-3095. doi:10.1096/fj.11-201681

16. Li X, Tan YC, Poulose S, Olanow CW, Huang XY, Yue Z (2007) Leucine-rich repeat kinase 2 (LRRK2)/PARK8 possesses GTPase activity that is altered in familial Parkinson's disease R1441C/G mutants. J Neurochem 103:238-247. doi:10.1111/j.1471-4159.2007.04743.x

17. Marder K, Wang Y, Alcalay RN, Mejia-Santana H, Tang MX, Lee A, Raymond D, Mirelman A, Saunders-Pullman R, Clark L et al (2015) Agespecific penetrance of LRRK2 G2019S in the Michael J. Fox Ashkenazi Jewish LRRK2 Consortium. Neurology 85:89-95. doi:10.1212/WNL. 0000000000001708

18. Moehle MS, Daher JP, Hull TD, Boddu R, Abdelmotilib HA, Mobley J, Kannarkat GT, Tansey MG, West AB (2015) The G2019S LRRK2 mutation increases myeloid cell chemotactic responses and enhances LRRK2 binding to actin-regulatory proteins. Hum Mol Genet 24:4250-4267. doi:10.1093/ hmg/ddv157

19. Nalls MA, Pankratz N, Lill CM, Do CB, Hernandez DG, Saad M, DeStefano AL, Kara E, Bras J, Sharma M et al (2014) Large-scale meta-analysis of genomewide association data identifies six new risk loci for Parkinson's disease. Nat Genet 46:989-993. doi:10.1038/ng.3043

20. Ozelius LJ, Senthil G, Saunders-Pullman R, Ohmann E, Deligtisch A, Tagliati M, Hunt AL, Klein C, Henick B, Hailpern SM et al (2006) LRRK2 G2019S as a cause of Parkinson's disease in Ashkenazi Jews. N Engl J Med 354:424-425. doi:10.1056/NEJMc055509

21. Paisan-Ruiz C, Jain S, Evans EW, Gilks WP, Simon J, van der Brug M, Lopez de Munain A, Aparicio S, Gil AM, Khan N et al (2004) Cloning of the gene containing mutations that cause PARK8-linked Parkinson's disease. Neuron 44:595-600. doi:10.1016/j.neuron.2004.10.023

22. Pisitkun T, Shen RF, Knepper MA (2004) Identification and proteomic profiling of exosomes in human urine. Proc Natl Acad Sci U S A 101:1336813373. doi:10.1073/pnas.0403453101

23. Sheng Z, Zhang S, Bustos D, Kleinheinz T, Le Pichon CE, Dominguez SL, Solanoy HO, Drummond J, Zhang X, Ding X et al (2012) Ser1292 autophosphorylation is an indicator of LRRK2 kinase activity and contributes to the cellular effects of PD mutations. Sci Transl Med 4:164ra161. doi:10.1126/scitranslmed.3004485

24. Skibinski G, Nakamura K, Cookson MR, Finkbeiner S (2014) Mutant LRRK2 toxicity in neurons depends on LRRK2 levels and synuclein but not kinase activity or inclusion bodies. J Neurosci 34:418-433. doi:10.1523/JNEUROSCI. 2712-13.2014

25. Smith WW, Pei Z, Jiang H, Dawson VL, Dawson TM, Ross CA (2006) Kinase activity of mutant LRRK2 mediates neuronal toxicity. Nature neuroscience 9: 1231-1233. doi: $10.1038 / \mathrm{nn} 1776$

26. Steger M, Tonelli F, Ito G, Davies $\mathrm{P}$, Trost M, Vetter M, Wachter S, Lorentzen E, Duddy G, Wilson Set al (2016) Phosphoproteomics reveals that Parkinson's disease kinase LRRK2 regulates a subset of Rab GTPases. Elife 5: doi:10.7554/eLife.12813

27. Street JM, Barran PE, Mackay CL, Weidt S, Balmforth C, Walsh TS, Chalmers RT, Webb DJ, Dear JW (2012) Identification and proteomic profiling of exosomes in human cerebrospinal fluid. J Transl Med 10:5. doi:10.1186/1479-5876-10-5

28. Thery C, Zitvogel L, Amigorena S (2002) Exosomes: composition, biogenesis and function. Nat Rev Immunol 2:569-579. doi:10.1038/nri855 
29. Trinh J, Guella I, Farrer MJ (2014) Disease penetrance of late-onset parkinsonism: a meta-analysis. JAMA Neurol 71:1535-1539. doi:10.1001/ jamaneurol.2014.1909

30. Vilas D, Shaw LM, Taylor P, Berg D, Brockmann K, Aasly J, Marras C, PontSunyer C, Rios J, Marek K et al (2016) Cerebrospinal fluid biomarkers and clinical features in leucine-rich repeat kinase 2 (LRRK2) mutation carriers. Mov Disord 31:906-914. doi:10.1002/mds.26591

31. Webber PJ, Smith AD, Sen S, Renfrow MB, Mobley JA, West AB (2011) Autophosphorylation in the leucine-rich repeat kinase 2 (LRRK2) GTPase domain modifies kinase and GTP-binding activities. J Mol Biol 412:94-110. doi:10.1016/j.jmb.2011.07.033

32. West $A B$ (2017) Achieving neuroprotection with LRRK2 kinase inhibitors in Parkinson disease. Exp Neurol: doi:10.1016/j.expneurol.2017.07.019

33. West AB, Moore DJ, Choi C, Andrabi SA, Li X, Dikeman D, Biskup S, Zhang Z, Lim KL, Dawson VL et al (2007) Parkinson's disease-associated mutations in LRRK2 link enhanced GTP-binding and kinase activities to neuronal toxicity. Hum Mol Genet 16:223-232. doi:10.1093/hmg/ddl471

34. Zimprich A, Biskup S, Leitner P, Lichtner P, Farrer M, Lincoln S, Kachergus J, Hulihan M, Uitti RJ, Calne DB et al (2004) Mutations in LRRK2 cause autosomal-dominant parkinsonism with pleomorphic pathology. Neuron 44: 601-607. doi:10.1016/.jneuron.2004.11.005

\section{Submit your next manuscript to BioMed Central} and we will help you at every step:

- We accept pre-submission inquiries

- Our selector tool helps you to find the most relevant journal

- We provide round the clock customer support

- Convenient online submission

- Thorough peer review

- Inclusion in PubMed and all major indexing services

- Maximum visibility for your research

Submit your manuscript at www.biomedcentral.com/submit 